\section{Remarque sur le problème de la mesure ${ }^{1}$ ).}

Par

\section{W. Sierpiński et E. Szpilrajn (Warszawa).}

Nous étudions dans la note présente les propriétés d'un certain ensemble indénombrable, en employant la notion de la convergence $(m)$, introduite récemment par M. F. Hausdorff ${ }^{2}$ ). Nous obtenons en même temps (sans faire l'usage de l'hypothèse du continu) une simple démonstration du théorème de MM. Banach, Kuratowski et Ulam, d'après lequel il n'existe, en dehors de la fonction identiquement nulle, aucune fonction d'ensemble, non négative (finie), complètement additive ${ }^{3}$ ) dans la famille de tous les sous-ensembles d'un ensemble de puissance $\aleph_{1}$ et qui s'annule pour les ensembles se réduisant à un point ${ }^{4}$ ) (III).

1. Admettons qu'une fonction non negative et finie $\mu(E) d \theta$ sous-ensemble d'un espace métrique $X$ est complètement additive, que la classe des ensembles pour lesquels $\mu$ est définie contient tous les sous-ensembles boreliens de $X$, que la classe des ensembles. sur lesquels $\mu$ s'annule est héréditaire ${ }^{5}$ ) et qu'elle contient tous les.

1) Présenté à la Société Polonajse de Mathématique (section de Varsovie), le 28. II. 1936

2) ce volume, p. 241.

3) Une fonction réelle $m(E)$ définie sur une classe $M$ d'ensembles s'appellecomplètement additive lorsqu'on a $m\left(E_{1}+E_{2}+\ldots\right)=m\left(E_{1}\right)+m\left(E_{2}\right)+\ldots$ pour chaque suite d'ensembles disjoints appartenant à $M$ et dont la somme appartient à $M$.

4) Cf. S. Banach et C. Kuratowski, Fund. Math. 14 (1929), p. 127; S. Clam, Fund. Math. 16 (1930), p. 140; W. Sierpiński: Hypothèse du con. tinu, Monogr. Mat. 4 (1934), pp. 44, 60, 107 et 159; E. Szpilrajn, Fund. Math. 22 (1934), p. 303. - M. Ulam a démontré ce théorème pour tous les alephs plus petits que le premier aleph inaccessible.

3) Une classe $\mathrm{K}$ d'ensembles s'appelle héreditaire lorsque chaque sous-ensemble d'un ensemble arbitraire appartenant à $K$ appartient également à $K$. ensembles se réduisant à un point. Nous appelons mesure dans $X$ chaque fonction $\mu$ remplissant ces conditions.

$N$ étant un soús-ensemble de $X$, considérons les propriétés suivantes:

(a) Chaque mesure dans $X$ s'annule sur $N$.

( $\beta)$ Chaque mesure dans $N$ s'annule sur $N$.

$(\gamma)$ Chaque mesure dans $N$, définie sur la classe de tous les sousensembles de $N$, s'annule sur $N$.

Il est évident que la propriété $(\gamma)$ peut être considérée pour un ensemble $N$ arbitraire (et non pas nécessairement métrique).

2. $X$ étant un espace métrique, une suite transfinie de sousensembles boreliens disjoints de $X$ :

$$
B_{0}, B_{1}, B_{2}, \ldots, B_{\xi}, \ldots
$$

s'appelle (d'après M. Hausđorff) convergente $(m)$, lọsqu'il existe pour chaque mesure $\mu(E)$ dans $X$ un nombre $\alpha<\Omega$ tel que

$$
\mu\left(\sum_{\alpha<<} B_{\xi}\right)=0 \text {. }
$$

Il existe, comme on sait (p. ex. dans l'espace $X$ des nombres réels), des suites $\left(^{*}\right)$ convergentes $(m)$ d'ensembles boreliens nonvides ${ }^{1}$ ). Soient donc: $p_{\xi}$ un point arbitraire de $B_{\xi}$ (pour chaque $\xi<\Omega$ ) et $N$ l'ensemble de tous les points $p_{\xi}$.

Or, l'ensemble $N$ jouit de la propriété $(\alpha)$. En effet, $\mu(E)$ étant une mesure dans $X$, il existe un nombre $\alpha<\Omega$ tel qu'on a l'égalité (:); il en résulte, en vertu de la relation

$$
\overline{\overline{N-\sum_{\tilde{\xi}<} \bar{B}_{\xi}}} \leqslant \aleph_{0}
$$

que $\mu(N)=0$.

Nous àvons ainsi démontré la proposition suivante:

I. Il existe (dans l'espace $X$ des nombres réels) ùn ensemble de puissance $\aleph_{1}$ qui jouit de la propriété $(\alpha)^{2}$ ).

1) Voir Hausd orff, l. c., p. 242.

2) L'existence d'un ensemble indénombrable qui jouit de la propriété (a) a été démontré pour la première fois par M. Poprougénko à l'aide de l'hy. pothèse du continu. Cf. Szpilrajn, l. c., pp. 311 et 307.

Fundamenta Mathematicae. T. XxvI. 
Toute décomposition d'un complémentaire analytique en constituantes disjointes étant convergente $(m)^{1}$ ), il en resulte qu'on obtient un ensemble linéaire de puissance $\aleph_{1}$ jouissant de la propriété $(\alpha)$, en décomposant un complémentaire analytique (linéaire) non borelien en constituantes disjointes et en choisissant un point de chaque constituante non vide. En particulier, on démontre qu'on peut prendre la décomposition connue de M. Lebesgue de l'in. tervalle en $\aleph_{1}$ ensembles non vides (dont la construction n'exige pas la connaissance de la théorie des ensembles analytiques) ${ }^{2}$ ).

3. Démontrons maintenant que

(i) La propriété $(\alpha)$ entraîne la propriété $(\beta)$.

Soient: $N$ un ensemble jouissant de la propriété $(\alpha)$ et $\mu(E)$ une mesure dans $N$, définie sur une classe $\boldsymbol{M}$ de sous-ensembles de $N$. Considérons la classe $M^{*}$ de tous les ensembles $M^{*}$ de la forme:

$$
M^{*}=M+E \quad \text { où } \quad M \in M \quad \text { et } \quad E \subset X-N \text {. }
$$

La classe $M^{*}$ contient tous les ensembles boreliens dans $X$ : en effet, $B$ étant un ensemble borelien dans $X$, on a

$$
\begin{gathered}
B=B N+B(X-N), \\
B N_{\epsilon} M, \quad B(X-N) \subset X-N ;
\end{gathered}
$$

l'ensemble $B$ est done de la forme (").

Posons pour chaque ensemble $M^{*}$ appartenant à $\mathbf{M}^{*}$, donc de la forme ("):

$$
\nu\left(M^{*}\right)=\mu(M) .
$$

La fonction $\nu$ est une mesure dans $X$ et on a

(i) $\nu(M)=\mu(M)$ pour chaque $M \in M^{*}$ tel que $M \subset N$.

L'ensemble $N$ jouissant de la propriété $(\alpha)$, on a $\nu(N)=0$, done - en vertu de $(i)-\mu(N)=0$. La proposition (i) est ainsi démontrée.

D'autre part il est clair que

(ii) La propriété $(\beta)$ entraîne la propriété $\left.(\gamma)^{3}\right)$.

(iii) La propriété $(\gamma)$ est un invariant par rapport aux transformations biunivoques (d'ensemble $N$ ).

1) Voir p.ex. E. S élivanow s ki, Fund. Math. 21 (1933), p. 20 (propriété $1^{0}$ ) qui a démontré aussi une propriété analogue des constituantes d'un ensemble analrtique lui-même (p. 24; ef. W. Sierpiński, Fund. Math. 21 (1933), p. 30). p. 209 .

2) Voir p.ex. W. Sierpiński, Leçons sur les nombres transfinis, Paris 1928,

3) Or, il est à remarquer que si $2^{N_{0}}=\aleph_{1}$ (ou même si $2^{\aleph_{0}}$ est plus petit que le premier aleph inaccessible), la propriété $(\gamma)$ n'entraine pas la propriété (j).

Les propositions (i)-(iii) entraînent le théorème suivant:

II. S'il existe (dans un espace métrique $X$ ) un ensemble $N$ de puissance it jouissant de la propriété $(\alpha)$, chaque ensemble de cette puissance jouit de la propriété $\left.(\gamma)^{1}\right)$.

Il résulte de I et II que

III. Chaque ensemble de puissance $\aleph_{1}$ jouit de la propriété $(\gamma)$.

4. Nous allons démontrer maintenant que

IV. Les propriétés $(\alpha)$ et $(\beta)$ sont équivalentes.

En vertu de $3(\mathrm{i})$, il reste à démontrer que la propriété $(\beta)$ entraîne la propriété $(\alpha)$.

Soient: $N \subset X$ un ensemble jouissant de la propriété $(\beta)$ et $\mu(E)$ une mesure dans $X$. Pour chaque ensemble $B$ borelien dans $N$, posons $\nu(B)$ égal à la borne inférieure de tous les nombres $\mu\left(B^{*}\right)$, où $B^{*}$ est un ensemble borelien dans $X$ et contenant $B$. Quand $\nu(B)=0$, posons encore $\nu(E)=0$ pour chaque ensemble $E \subset B$.

Montrons que la fonction $\nu$ est complètement additive dans la classe des ensembles boreliens dans $N$; soit donc

$$
B=B_{1}+B_{2}+B_{3}+\ldots
$$

une série d'ensembles disjoints, boreliens dans $N$. Par conséquent il existe une suite $\left\{B_{n}^{*}\right\}$ d'ensembles disjoints, boreliens dans $X$, tels que $N B_{n}^{*}=B_{n}$ pour $n=1,2, \ldots$ Posons:

$$
B^{*}=B_{1}^{*}+B_{2}^{*}+B_{3}^{*}+\ldots
$$

Soit $\varepsilon$ un nombre positif arbitraire. II existe un ensemble $B^{0}$ borelien dans $X$, tel que

$(\%)$

et

$$
v(B)+\varepsilon>\mu\left(B^{0}\right)
$$

On a, en vertu de (j): $B^{0} B_{n}^{*} \supset B_{n}$ et par conséquent

$$
\mu\left(B^{0}\right)=\mu\left(B^{0} B_{1}^{*}\right)+\mu\left(B^{0} B_{2}^{*}\right)+\ldots \geqslant \nu\left(B_{1}\right)+\nu\left(B_{2}\right)+\ldots
$$

donc, d'après (:):

$$
\nu(B)+\varepsilon \geqslant \nu\left(B_{1}\right)+\nu\left(B_{2}\right)+\ldots
$$

1) Cf. Szpilrajn, l. c., p. 308 . 
D'autre part, il existe (pour $n=1,2, \ldots$ ) un ensemble $A_{n} \supset B_{n}$, borelien dans $N$ et tel que

$$
\mu\left(A_{n}\right) \leqslant \nu\left(B_{n}\right)+\frac{\varepsilon}{2^{n}} .
$$

En posant $A=A_{1}+A_{2}+\ldots$, nous obtenons

(菜) $) \quad \nu(B) \leqslant \mu(A) \leqslant \mu\left(A_{1}\right)+\mu\left(A_{2}\right)+\ldots \leqslant \varepsilon+\nu\left(B_{1}\right)+\nu\left(B_{2}\right)+\ldots$

$\varepsilon$ étant un nombre positif arbitraire, il résulte de $(\%)$ et (\%) que

$$
\nu(B)=\nu\left(B_{1}\right)+\nu\left(B_{2}\right)+\ldots,
$$

ce qui démontre l'additivité complète de la fonction $\nu$.

Dès lors, il est clair que la fonction $\nu$ est une mesure dans $N$. L'ensemble $N$ jouissant de la propriété $(\beta)$, on a $\nu(N)=0$. Il résulte de la définition de la fonction $\nu$ qu'il existe un ensemble $B$ borelien dans $X$, contenant $N$ et tel que $\mu(B)=\nu(N)$. Par conséquent, $\mu(N)=0$, c. q. f. d.

5. Remarquons maintenant que chaque transformation biunivoque mesurable $(B)$ de l'espace $Z$ en $Z^{*}$ transforme chaque mesure dans $Z$ en une mesure dans $Z^{*}$ ( $Z$ et $Z^{*}$ étant supposés séparables). En d'autres mots:

(o) Soient: $\mu(E)$ une mesure dans $Z$ définie sur une classe $\boldsymbol{M}$ de sous-ensembles de $Z, f$ une transformation biunivoque mesurable $(B)$ de $Z$ en $Z^{*}$ et $M^{*}$ la classe de tous les ensembles $f(M)$, où $M \in M$. Thèse: La fonction $\mu\left(f^{-1}(E)\right)$, définie pour chaque $E \in M^{*}$, est une mesure dans $Z^{*}$.

Pour la démonstration, il suffit de remarquer que l'ensemble $f^{-1}(B)$ étant borelien dans $Z$ pour chaque ensemble $B$ borelien dans $Z^{*}$, la classe $M^{*}$ contient tous les ensembles boreliens dans $Z^{*}$.

La proposition (o) permet de démontrer que dans le domaine des ensembles séparables:

V. La propriéte $(\beta)$ est invariante par rapport aux transformations biunivoques, inverses aux transformations mesurables $(B)$.

Soient: $N_{1}$ et $N_{2}$ deux ensembles métriques séparables dont le premier jouit de la propriété $(\beta)$ et $\varphi(x)$ une transformation biunivoque de $N_{1}$ en $N_{2}$ dont l'inversion est mesurable $(B)$. Désignons par $\mu(E)$ une mesure arbitraire dans $N_{2}$, par $M$ la classe des ensembles sur laquelle elle est définie, et par $M *$ la classe de tous les ensembles $\varphi^{-1}(M)$, où $M \in M$. En vertu de (o), la fonction $\mu(\varphi(E))$, définie pour chaque $E \in \mathrm{M}^{*}$, est une mesure dans $N_{1}$, on a donc $\boldsymbol{\mu}\left(\varphi\left(N_{1}\right)\right)=0$ et, en d'autres mots, $\boldsymbol{\mu}\left(N_{2}\right)=0$, c. q. f. d.

Il est à remarquer que chaque ensemble séparable $K$ de.puissance $\aleph_{1}$ est une image biunivoque et continue d'un ensemble' jouissant de la propriété ( $\beta$ ). (Soient $N$ un ensemble séparable de puissance $\aleph_{1}$ jouissant de la propriété $(\beta), \psi$ une transformation biunivoque de $N$ en $K$ et $I$ l'image de la fonction $\psi$. L'ensemble $K$ est une image continue et biunivoque de l'ensemble $I$.qui jouit - en vertu de $\mathrm{V}$ - de la propriété $(\beta)$ ). Par conséquent, si $2{ }^{*}=\aleph_{1}$, la propriété $(\beta)$ n'est pas invariante par rapport aux transformations biunivoques et continues.

\section{II résulte de $I, I V$ et $V$ que}

VI. Il existe un ensemble linéaire indénombrable dont chaque image linéaire obtenue par une transformation biunivoque; inverse à une transformation mesurable $(B)$, est de mesure lebesguienne nulle ${ }^{1}$ ).

1) En particulier, chaque image de cet ensemble obtenue par une homéomorphie généralisée est de mesure nulle. Cf. W. Sierpiński, Fund. Math. 7 (1925), p. 188 et Publ. Math. Univ. Belgrade 2 (1933), p. 19. 